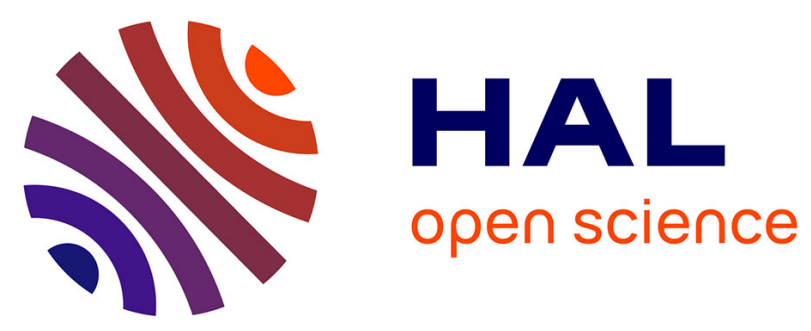

\title{
Hagiographie et diplomatique dans le monachisme réformé en Bourgogne au miroir du manuscrit 1 de Semur-en-Auxois
}

Eliana Magnani

\section{- To cite this version:}

Eliana Magnani. Hagiographie et diplomatique dans le monachisme réformé en Bourgogne au miroir du manuscrit 1 de Semur-en-Auxois. Marie-Céline Isaïa; Thomas Granier. Normes et hagiographie dans l'Occident latin (VIe-XVIe siècle). Actes du colloque international de Lyon, 4-6 octobre 2010, 9, Brepols, pp.183-195, 2014, Hagiologia, 978-2-503-54835-7. halshs-01097486

\section{HAL Id: halshs-01097486 \\ https://shs.hal.science/halshs-01097486}

Submitted on 19 Dec 2014

HAL is a multi-disciplinary open access archive for the deposit and dissemination of scientific research documents, whether they are published or not. The documents may come from teaching and research institutions in France or abroad, or from public or private research centers.
L'archive ouverte pluridisciplinaire HAL, est destinée au dépôt et à la diffusion de documents scientifiques de niveau recherche, publiés ou non, émanant des établissements d'enseignement et de recherche français ou étrangers, des laboratoires publics ou privés.

\section{(1) (1) $\$$}

Distributed under a Creative Commons Attribution - NonCommercial - NoDerivatives 44.0 


\title{
Hagiographie et diplomatique dans le monachisme réformé en Bourgogne au miroir du manuscrit 1 de Semur-en-Auxois
}

\author{
Eliana MAGNANI
}

(paru dans Normes et hagiographie dans l'Occident latin (VI $I^{e} X V I^{e}$ siècles), éd. par MarieCéline IsAÏA et Thomas GARNIER, Turnhout, Brepols, 2014 (Hagiologia, 9), p. 183-195)

Le propos de cet article est, à partir de l'exemple du manuscrit 1 de la Bibliothèque municipale de Semur-en-Auxois ${ }^{1}$, de penser la question de la transtextualité, en empruntant cette notion à la théorie littéraire ${ }^{2}$, tout en la restituant non seulement en fonction du texte de l'énoncé, mais également en relation aux signes visuels et matériels de la mise par écrit, "la raison graphique »" sur la longue durée. Il s'agit aussi d'interroger le rôle des différents chantiers scripturaires propres à la renovatio monastique au tournant des $\mathrm{X}^{\mathrm{e}}$ et $\mathrm{XI}^{\mathrm{e}}$ siècles, comme l'un des moyens de «mise en norme » dans le cadre d'une redéfinition plus large de la place occupée par les monastères dans la société seigneuriale.

\section{Le manuscrit}

Tel qu'il se présente aujourd'hui, dans sa reliure du XIX ${ }^{\mathrm{e}}$ siècle, le manuscrit 1 de Semur, provenant de l'abbaye Saint-Jean de Réôme (Moutiers-Saint-Jean), en Côted'Or, contient des pièces allant des alentours de l'an mil jusqu'au $X V^{\mathrm{e}}$ siècle, ainsi que des annotations des époques moderne et contemporaine. De taille moyenne $(260 \mathrm{x}$ $207 \mathrm{~mm}$ ), comportant 78 feuillets, il est composé du livret hagio-liturgique de saint Jean de Réôme, fondateur et premier abbé de l'abbaye de Réôme (vers 470), réalisé vraisemblablement entre la fin du $\mathrm{X}^{\mathrm{e}}$ et le début du XI $\mathrm{XI}^{\mathrm{e}}$ siècle [fol. 0r-69v], suivi de deux célèbres diplômes mérovingiens en faveur de l'abbaye, de Clovis $\mathrm{I}^{\mathrm{er}}$ (481/82-511; diplôme de 498, le 29 décembre, à Reims) [fol. 71v-72v] et de Clotaire I ${ }^{\text {er }}$ (511-561; diplôme de 516, le 22 février, à Soissons) [fol. 73r], forgés respectivement, selon Theo

\footnotetext{
${ }^{1}$ Ce travail est issu de la recherche collective réalisée dans le cadre des séminaires de master 2 à l’Université de Bourgogne, sur le manuscrit 1 de la Bibliothèque municipale de Semur-en-Auxois, en collaboration avec Daniel Russo et Eduardo H. Aubert, et avec le soutien de M. Jean-Claude Sosnowski, directeur de la bibliothèque de Semur-en-Auxois. En attendant la publication en cours de cette enquête, on peut se rapporter aux articles suivants: D. RUSSO, "Étude sur le manuscrit 1 de la Bibliothèque municipale de Semur-en-Auxois : enluminure de manuscrit et réforme monastique. ", Bulletin du Centre d'études médiévales d'Auxerre, 10 (2006), [en ligne] http://cem.revues.org/document368.html ; D. RUSSO, E. MAGNANI, « Le manuscrit 1 de la Bibliothèque municipale de Semur-en-Auxois, provenant de l'abbaye de Saint-Jean de Réome (Moutiers-Saint-Jean): programme pédagogique de recherche », Bulletin du Centre d'études médiévales d'Auxerre, 12 (2008), [en ligne] http://cem.revues.org/document7212.html; E. H. AuberT, E. MAGNANI, D. Russo, « Histoire du manuscrit médiéval, transmission des textes, des chants, compositions peintes et décors. », Bulletin du centre d'études médiévales d'Auxerre, 13 (2009), [en ligne] http://cem.revues.org/index11055.html ; E. H. Aubert, E.Magnani, D. Russo, «Le manuscrit 1 de Semur-en-Auxois », BUCEMA -Bulletin du centre d'études médiévales d'Auxerre, 14 (2010), p. 101-112, [en ligne] http://cem.revues.org/index11561.html

${ }^{2}$ En particulier, G. GeneTte, Palimpsestes, Paris, 1982.

${ }^{3}$ Il s'agit ici, bien entendu, d'une référence aux travaux de Jack Goody sur la literacy (J. GoODY, La raison graphique. La domestication de la pensée sauvage, trad. et présentation de J. Bazin et A. Bensa, Paris, 1979 (The Domestication of the Savage Mind, Cambridge, 1977)) ; pour la mise au point de la recherche dans ce domaine, en ce qui concerne le Moyen Âge, voir P. CHASTANG, «L'archéologie du texte médiéval. Autour de travaux récents sur l'écrit au Moyen Âge », Annales HSS, (Mars-Avril 2008), p. 245-269.
} 
Kölzer, leur plus récent éditeur, au début et dans la seconde moitié du XI $\mathrm{XI}^{\mathrm{e}}$ siècle $^{4}$. Dans la page restée en blanc du bifeuillet du diplôme de Clovis [fol.71v], une main probablement $\mathrm{du} \mathrm{XIII}^{\mathrm{e}}$ siècle y a transcrit une liste de redevances (cruces) dues à l'abbaye de Réôme par ses églises dépendantes. Le verso du feuillet contenant le diplôme de Clotaire [fol.73v], laissé également en blanc, porte des notes d'une main du $\mathrm{XIV}^{\mathrm{e}}$ siècle qui a écrit le catalogue des évêques de Langres [fol. 74r] et peut-être aussi la liste des abbés de Réôme [fol. 75v-76v] ${ }^{5}$. Cette main (ou l'une de ces mains du XIV siècle) a probablement annoté les diplômes [fol.72v et $73 \mathrm{r}$ ] et le livret hagiographique [fol. 55v et 69r]. Les indices codicologiques indiquent que ces éléments - composition hagio-liturgique, diplômes royaux, catalogues - étaient réunis au plus tard au milieu du $\mathrm{XIV}^{\mathrm{e}}$ siècle, au moment de la réalisation de la liste des évêques et des abbés. En revanche, il n'est pas aisé de savoir si les diplômes royaux étaient reliés matériellement au livret hagiographique plus tôt, malgré l'intertextualité des deux productions. L'absence d'un feuillet à la fin du livret hagiographique, ainsi que les marques de dégradation des trois premiers feuillets (aujourd'hui restaurés) tendent cependant à indiquer qu'il a été conservé longtemps indépendamment et sans reliure ${ }^{6}$.

\section{Le libellus de saint Jean de Réôme et les diplômes royaux}

Le livret hagio-liturgique de saint Jean de Réôme est le résultat d'un projet unitaire, exécuté peut-être en différentes campagnes proches dans le temps, de création textuelle, musicale et plastique. La première partie, proprement liturgique, est constituée des lectures pour l'office de matines de l'anniversaire du saint (28 janvier) (BHL 4425, livre 1 ; $B H L$ 4426, miracles) [fol. 0v-15r], suivies des lectures pour la fête de la translation (22 septembre) issues, pour la plupart de la vie ( $B H L 4425$, livre 1), des miracles $(B H L$ 4426), de la passion de Maurice d'Agaune et de ses compagnons (BHL 5741) et des translations de Jean $(B H L 4429,4430)$ [fol. 15v-45v]. Chaque leçon est marquée par des initiales finement enluminées [entre fol. 2r-38r, 23 lettrines], tandis que des doubles pages monumentales marquent l'ouverture des deux fêtes [fol. $0 \mathrm{v}-1 \mathrm{r}$ et $15 \mathrm{v}-16 \mathrm{r}$ ]. Les notes marginales contemporaines de révision du découpage du texte en leçons [entre les fol. $20 \mathrm{v}$ et $43 \mathrm{v}$ ], indiquent que l'office de la translation était une nouveauté dont le manuscrit témoigne de la mise en place. Ensuite ont été transcrites des pièces chantées propres pour les offices de vêpres, laudes et matines, dont plusieurs portent des neumes [fol. 45v-50r]. Dans la deuxième partie du livret, la version BHL 4426 de la vie de Jean

\footnotetext{
${ }^{4}$ Die Urkunden der Merowinger, éd. préparée par C. BRÜHL, publiée par T. KöLZER avec le concours de M. Hartmann et A. Stieldorf, 2 vol., Hanovre, 2001 [MGH. Diplomata regum Francorum e stirpe merovingica], t. 1 ( $\mathrm{n}^{\circ} 3$, p. 7-10; $\mathrm{n}^{\circ} 15$ p. 47-49). Voir aussi les hypothèses de C. BRÜHL, « Clovis chez les faussaires », Bibliothèque de l'Ecole des Chartes, 154 (1996), p. 219-240, en particulier p. 234-237 et ID., Studien zu den merowingischen Königsurkunden, éd. T. KöLZER, Cologne-Weimar-Vienne, 1998, p. 75 ss., ainsi que C. B. Bouchard, " High Medieval Monks Contemplate their Merovingian Past », The Journal of Medieval Monastic Studies, 1 (2012), p. 41-62, en particulier p. 44-46.

${ }^{5}$ Sur le verso du feuillet 74, est mentionnée une liste des officiers du monastère, datant peut-être du XIII ${ }^{\mathrm{e}}$ siècle. Une charte-partie datant de 1182 à été ajoutée à l'ensemble lors de la nouvelle reliure réalisée après 1834 (fol. 77r). Le feuillet 70 disparaît à ce moment; il est signalé dans le catalogue manuscrit de 1834 comme « garde tirée d'un vieux rituel ». Le manuscrit se termine par un feuillet (fol. 78) contenant des notes $\mathrm{du} \mathrm{XV}^{\mathrm{e}}$ siècle, dont le blason de l'abbé Jean II de Cussigny (1476-1491) et l'inscription de la date «l'an mil quatre cents cinquante trois le jour de $[\ldots] »$.

${ }^{6}$ Sur les caractéristiques codicologiques récurrentes des livrets hagiographiques et la bibliographie sur ce type de manuscrit, voir J.-Cl. POULIN, «Les libelli dans l'édition hagiographique avant le XII ${ }^{\mathrm{e}}$ siècle », dans Livrets, collections et textes. Etudes sur la tradition hagiographique latine, dir. M. HEINZELMANN, Ostfildern, 2006 (Beihefte der Francia 63), p. 15-193, ainsi que l'article précurseur de Francis Wormald sur les livrets hagiographiques enluminés (F. WORMALD, « Some illustreted manuscripts of the live of the saints », Bulletin of The John Rylands Library (Manchester), 35 (1952-53), p. 248-266).
} 
de Réôme a été copiée. Les deux livres de la $B H L 4426$ sont introduits par deux lettres monumentales [fol. $52 \mathrm{v} ; 63 \mathrm{v}$ ], alors que chaque chapitre débute par une initiale et une rubrique en capitales rouges ou noires, à l'exception de la lettrine peinte du feuillet $69 \mathrm{r}^{7}$. Selon Bruno Krusch, éditeur de la vie de Jean de Réôme écrite par Jonas de Bobbio (vers 659) (BHL 4424 ; « version A »), le premier livre de la BHL 4425 (qu'il appelle « version B ») ${ }^{8}$ et la $B H L 4426$ (« version C », en deux livres) auraient été composés au monastère de Réôme à l'époque carolingienne, respectivement à l'époque de Charlemagne et au IX ${ }^{\mathrm{e}}$ siècle (avant 888$)^{9}$.

Malgré la qualité de la réalisation du livret hagiographique, ce sont les deux diplômes royaux qui ont fait la notoriété du manuscrit, depuis surtout leur édition en 1637 par Pierre Rouvier ${ }^{10}$. En page d'ouverture, une main moderne a noté : «à la page 72 du présent livre sont les chartres de la fondation de nostre Abbaye par clovis premier, Roy chrétien, et confirmées par clotaire son fils en $516 »$. Cependant c'est le récit hagiographique qui semble être l'une des sources utilisées par les créateurs des diplômes qui puisent notamment à des formules carolingiennes ${ }^{11}$. Le choix même des deux rois, Clovis et Clotaire, paraît provenir de leur mention dans les versions carolingiennes de la vie de Jean de Réôme, qui figurent dans le manuscrit 1 de Semuren-Auxois. Les BHL 4425 et 4426 citent Clovis en début de récit, tandis que la $B H L$ 4426 est la seule à citer Clotaire, à la fin, au dernier chapitre du deuxième livre. Dans le cas de Clovis, les versions carolingiennes s'inspirent sans doute de la mention « furtive » du roi dans le chapitre 15 de la vie de Jean de Réôme par Jonas de Bobbio pour situer un miracle à l'époque de la campagne en Italie de son petit-fils Théodebert

\footnotetext{
${ }^{7}$ Le livret est amputé d'un feuillet au moins à la fin. Il manque aussi un feuillet entre les fol. 5 et 6 , et deux entre les fol. 17 et 18 . Le livret est constitué de dix cahiers signés de A à $\mathrm{K}$; il s'agit de quaternions, sauf le cahier $\mathrm{G}$, qui est un ternion. Les compositions versifiées des feuillets $0 \mathrm{r}$ et $15 \mathrm{r}$ (BHL 4428a et 4428b), ainsi que deux prières du fol. 50r, ont été insérées au XII siècle. Le contenu des feuillets manquants du livret hagiographique du manuscrit 1 de Semur-en-Auxois peut être restitué grâce au manuscrit de la Bibliothèque Apostolique Vaticane, Reg. Lat. 493, fol. 105r-135v, pour lequel il a servi d'hypotexte.

${ }^{8}$ Selon les Bollandistes, la $B H L 4425$ est composée en deux livres, le deuxième étant identique à celui de la $B H L$ 4426. Il s'agit, en fait, du montage donné par l'édition de Mabillon [Acta Sanctorum ordinis $S$. Benedicti in Saeculorum Classes distributa. Saeculum I., Paris, 1668, p. 632-636 (BHL 4425, livre 1), p. 637-639 (BHL 4426, livre 2), p. 639-642 (BHL 4429)].

9 Jonas de Bobbio, Vita Ihoannis abbatis Reomaensis autore Ionas, éd. B. KRUSCH, Hannovre, 1896 (MGH Scriptores rerum merovingicarum, III), p. 505-517, IBID., Hannovre, 1905 (MGH, SS rer. Germ. in usum scholarum), p. 321-344; B. KRUSCH, «Zwei Heiligenleben des Jonas von Susa. I : Die Vita Johannis Reomaensis », Mitteilungen des Instituts für österreichische Geschichtsforschung, 14 (1893), p. 385-427. Voir à propos de la vie de Jean de Réome par Jonas de Bobbio (BHL 4424) : J. MARILIER, "Le monastère de Moutier-Saint-Jean et ses attaches colombaniennes », dans Mélanges colombaniens, actes du congrès international de Luxeuil, 20-23 juillet 1950, Paris, 1951, p. 383-384; ID., "Notes d'hagiographie lingone (Guillaume de Dijon ou de Volpiano, saint Jean de Réome », Bulletin de la Société historique et archéologique de Langres, 16/224-25 (1971), p. 121-122, A. DUBREUCQ, « Lérins et la Burgondie dans le haut Moyen Âge », dans Lérins, une île sainte de l'Antiquité au Moyen Âge, éds. Y. Codou, M. Lauwers, Turnhout, 2009, p. 195-227 (en particulier p. 206-212) ; A. DiEM, « The Rule of an 'Iro-Egyptian' monk in Gaul Jonas' Vita Iohannis and the construction of a monastic identity », Revue Mabillon, 19 (2008), p. 5-50.

10 P. Rouvier, (S.J., Le P.), Reomaus, seu Historia monasterii S. Joannis Reomaensis, in tractu Lingonensi, primariae inter gallica coenobia antiquitatis, ab anno Christi 425 , collecta et illustrata a $P$. Petro Roverio..., Paris, 1637, p. 28-30, 71-72. Rouvier est également le premier éditeur des $B H L 4426$ (p. 1-23 et 70-71), BHL 4429 (p. 40-47 et extrait p. 73-74), BHL 4430 (p. 47-57) et BHL 4431 (p. 57-58). Sur les débats autour de l'authenticité des diplômes de Clovis et Clotaire, outre l'édition de Theo Kölzer, voir A. RAuwel, "La fausse charte de Clovis pour Moutiers-Saint-Jean », Bulletin de l'Académie des Sciences et des Lettres de Semur (2005), p. 66-71.

${ }^{11}$ Die Urkunden der Merowinger, op. cit., t. 1, p. 8.
} 
$(533-547 / 48)^{12}$. Dans les versions carolingiennes, ce repère sert à localiser la durée de vie de saint Jean dans la succession des empereurs romains et des rois des Francs : de Clovis à Théodebert, en passant par Théodéric (511-533) ${ }^{13}$, rois cités à nouveau dans le récit de miracle du deuxième livre de la $B H L 4426^{14}$. Clotaire $\mathrm{I}^{\mathrm{er}}$, en revanche, ne figure pas dans le récit composé par Jonas de Bobbio. Il est une «nouveauté » apportée par le dernier chapitre de la $B H L 4426$ qui rend compte de la succession de saint Jean et de la continuité de son œuvre, par l'abbé Silvestre. Sur celui-ci l'hagiographe note l'attachement que lui ont voué les rois des Francs Clotaire et Childebert (511-558) et rapporte un miracle connu par le récit de Venance Fortunat (v. 530-609) dans la vie de saint Germain de Paris (496-576) (BHL 3468, chapitre 35) ${ }^{15}$.

Bien que très allusives, il n'en reste pas moins que ces mentions placent clairement les deux premiers abbés de Réôme en parallèle avec le règne des premiers mérovingiens en Gaule. Les « rois des Francs » sont aussi évoqués de manière générique au chapitre 10 du deuxième livre de la $B H L$ 4426. Mais ici ils sont signalés, avec d'autres nobles, comme ayant agi directement en faveur du monastère de Réôme, puisqu'on peut, dit-on, toujours relire les chartes conservées dans les archives, par lesquelles ils ont concédé leurs bénéfices ${ }^{16}$. La façon dont ce passage de la vie se présente aujourd'hui dans le feuillet 69r du manuscrit 1 de Semur-en-Auxois pourrait indiquer l'attention qu'on lui a

\footnotetext{
${ }^{12}$ Jonas de Bobbio, Vita Ihoannis abbatis, op. cit., p. 513 , p. 338 (Cumque iam Gallias Francorum regis sue dictione, sublato imperii iure, gubernacula ponerent et, postposita rei publice dominatione, propria fruerentur potestate, evenit, ut Theudebertus, filius Teuderici, Clodovei condam filii, bellum Italie inferret, transactis Alpibus, Italiam inquietaret). Voir M. HEINZELMANN, "Clovis dans le discours hagiographique du VI ${ }^{\mathrm{e}}$ au IX $\mathrm{e}^{\mathrm{e}}$ siècle », Bibliothèque de l'Ecole des Chartes, 154 (1996), p. 87-112 (ici, et pour l'expression « furtive », p. 102, n. 66).

${ }^{13}$ [BHL 4425] : fol. 2r-v (lectio secunda) : Quo etiam tempore, franci cum Chlodoueo rege post posta republica, militari manu terminos romanorum irrumpentes, Galliam inuaserunt. Sicque usque in tempora Theoderici regis qui filius extitit prefati Chlodouei, et filii eius Theodeberti perdurauit. Nam quando sacris artius se mancipauit documentis, Gallias sub imperii iure, Iohannes consul regebat; fol. 17v (lectio secunda) : Quo etiam tempore, franci cum Chlodoueo rege, post posita republica, militari manu terminos romanorum irrumpentes, Galliam inuaserunt. Sicque, usque in tempora Theoderici perdurauit. Nam quando sacris artius se mancipauit documentis, Gallias sub imperii iure, Iohannes consul regebat ; [BHL 4426] : fol. 51v (praefatio) : Quo etiam tempore, franci cum Clodoueo rege, post posita republica, militari manu terminos romanorum irrumpentes, Galliam inuaserunt. Sicque usque in tempora Theoderici regis, qui filius extitit prefati Clodouei, et filii eius Theodeberti perdurauit. His itaque omissis, ad ea que coepimus reuertamur.

${ }^{14}$ [BHL 4426] : fol. 10r : Nam tempore quo franci post posita republica, sublatoque imperii iure, propria dominabantur potestate, Theodebertus filius Theuderici, Chlodouei quondam filii, Italie claustris disruptis, bellum Ausoniis intulit; fol. 65v : Nam tempore quo franci post posita re publica, sublatoque imperii iure, propria dominabantur potestate, Theodebertus filius Theuderici, Clodouei quondam filii, Italiae claustris disruptis, bellum Ausoniis intulit.

${ }^{15}$ Le passage ne figure plus dans le manuscrit 1 de Semur, qui a perdu ses derniers feuillets, mais le chapitre correspondant est cité dans la table des chapitres du deuxième livre (f. 60r - XII De praelatione abbatis SILVESTRI sicut ipsus beatus iohannes adhuc uiuentis praecepit). Le texte du chapitre peut être reconstitué grâce au manuscrit Reg. lat. 493, f. 135v et l'édition de Pierre Rouvier, Reomaus, op. cit., p. 70 : Post obitum gloriosissimi domini Ihoannis, suffectus est in locum eius abbas Silvester nomine quem ipse antea uiuens fratrum coetui praesse praeceperat; qui et religionis forma et regulae tenore per vestigia magistri gradiens longevo floruit tempore. Diligebatur autem a Chlotario atque Childberto christianissimis regibus francorum ob meritum eius sanctitatis.)

${ }^{16}$ fol. $69 \mathrm{r}$ : QVANTO IAM HONORE AC VENERATIONE regum francorum atque nobilium fulciretur uirorum, ambigit nemo, qui beneficia a predictis regibus prestita, per precepta chartarum que usque nunc in publicis arciuis predicti condita sunt monasterii, relegere cupit. Le rapprochement entre ce passage de la BHL 4426 et les diplômes de Clovis et Clotaire a été fait par B. Krusch (MGH Scriptores rerum merovingicarum, III, op. cit., p. 504 ; ID., «Zwei Heiligenleben... », op. cit., p. 407 ; ID., " Studien zur fränkischen Diplomatik. Der Titel der fränkischen Könige », Abhandlungen der Preussischen Akademie der Wissenschaften, Philosophisch-historische Klasse, 1 (1937), p. 44), et discuté aussi par C. Bruhl et T. Kölzer (voir références supra n. 4).
} 
apportée depuis longtemps. Ainsi, la note marginale d'une main du XIV ${ }^{\mathrm{e}}$ siècle qu'on lit partiellement, [...] infra [...] folio, marque sans doute le renvoi vers les actes de Clovis et Clotaire ajoutés à la suite du livret hagiographique de saint Jean. Plus encore, le passage est mis en relief par le traitement différencié donné à la lettre « Q " (Quantum) au début du chapitre 10 : contour noir appuyé, et intérieur du cercle partagé en quatre champs peints en rouge, bleu et jaune (fig. 1). Effectivement, à l'inverse des leçons de l'office qui dans le manuscrit commencent toutes par des lettrines finement enluminées (fig. 2 et 3), dans la transcription de la BHL 4426 seul le début des deux livres portent une grande initiale décorée. Tous les chapitres sont marqués, quant à eux, par le traitement rubriqué de la première ligne, en rouge ou en noir: une première lettre majuscule grasse sur deux lignes suivie de capitales (fig. 4). Il n'est pas possible de déterminer avec certitude si l'initiale « $\mathrm{Q}$ » du feuillet $69 \mathrm{r}$ a été peinte au moment de la réalisation du livret hagiographique, ou s'il s'agit d'une intervention postérieure, ce qui semble cependant une hypothèse assez plausible. Mais quelle que soit l'époque, l'ajout des couleurs dans une initiale à l'intérieur d'une partie du manuscrit où ce procédé n'est pas utilisé, signale l'intention d'attirer le regard sur ce passage du texte en particulier. Qui plus est, le type de peinture choisie pour le « $\mathrm{Q}$ », quatre triangles colorés, est le même que celui utilisé dans la première lettrine du livret hagiographique, le « $\mathrm{D}$ » (Denique) (fig. 2), qui introduit la lectio secunda de la fête de l'anniversaire de saint Jean, et où se trouve en l'occurrence la première mention de Clovis, de Théodéric et de Théodebert dans le manuscrit. Ces marquages visuels semblent indiquer la volonté de faire résonner les différentes parties du livret hagio-liturgique avec les chartes des rois des Francs ; ils indiquent la mise en relation volontaire de ces différentes réalisations.

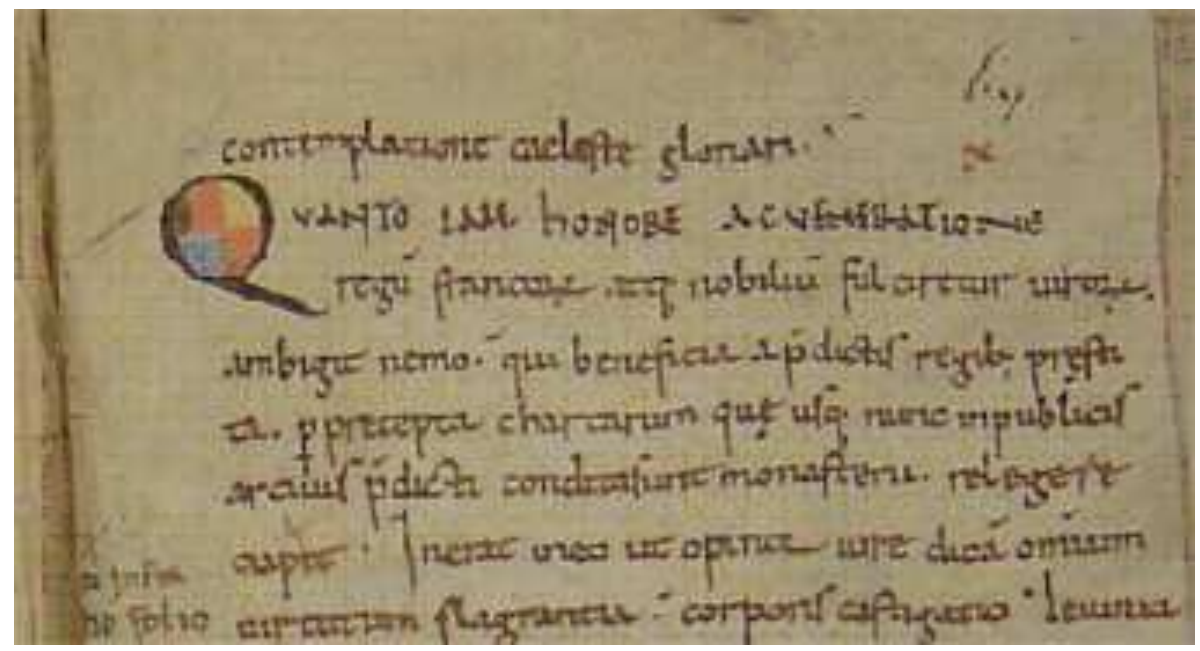

Fig. 1 : Semur-en-Auxois, Bibliothèque municipale, ms. 1, fol. 69r

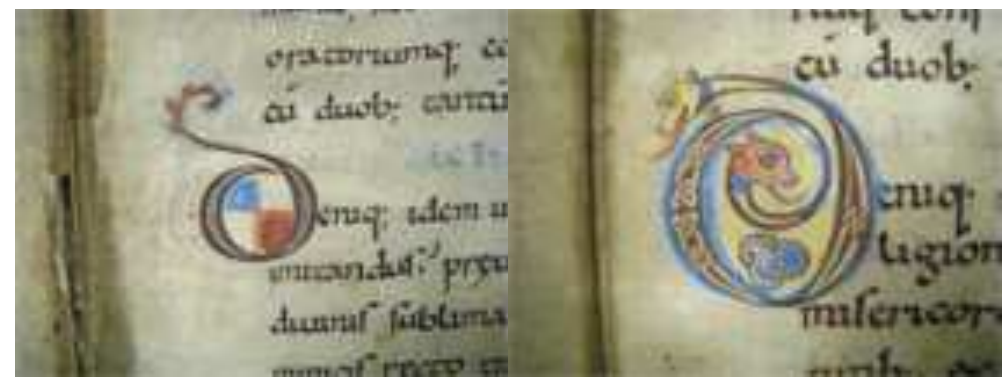

Fig. 2 et 3 : Semur-en-Auxois, Bibliothèque municipale, ms. 1, fol. $2 \mathrm{r}$ et $17 \mathrm{r}$ 


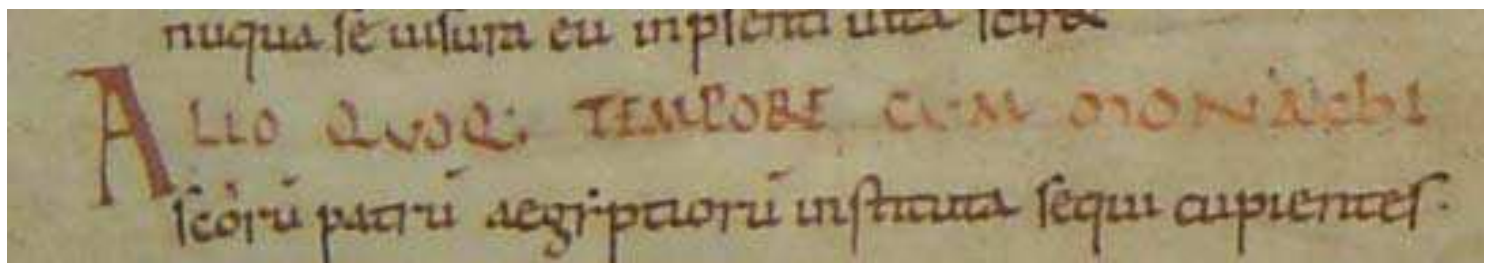

Fig. 4 : Semur-en-Auxois, Bibliothèque municipale, ms. 1, fol. 58v

\section{Intertextualités}

Dans le diplôme de Clovis - qui prend le monastère de Réôme sous sa protection, lui confère des biens et l'immunité -, plusieurs éléments prolongent cette résonance du point de vue de l'intertextualité avec les récits hagiographiques et les différentes pièces liturgiques connues grâce au manuscrit 1 de Semur-en-Auxois. L'évocation, dans le diplôme, de l'année de la « soumission des Gaulois » par Clovis est probablement un rappel de «l'invasion de la Gaule » par Clovis dans la lectio secunda de la BHL 4425 et dans la préface de la $B H L 4426$, augmenté de sa conversion au christianisme ${ }^{17}$. La façon de désigner Jean, à cinq reprises, dans ce même diplôme, comme patronus - mot utilisé dans les actes diplomatiques pour désigner un saint patron, mais pas un abbé vivant ${ }^{18}$ renvoie à l'usage du vocable dans les textes liturgiques chantés lors des offices consacrés à saint Jean. Dans le manuscrit 1, cette épithète est employée dans une homélie, dans une oraison, dans un répons, dans un hymne et dans un passage de la $B H L 4426$ qui fait justement référence à la louange qui est due au saint ${ }^{19}$. Le diplôme de Clovis fait aussi référence à la règle de Macaire que Jean introduit dans son monastère ${ }^{20}$, donnée qui se trouve dans les différentes versions de la vie de Jean ${ }^{21}$.

Le diplôme dialogue aussi avec d'autres récits hagiographiques. La manière de délimiter le domaine que le roi octroie à Réôme, c'est-à-dire, le circuit parcouru autour du monastère sur un âne au long d'une journée ${ }^{22}$, évoque un procédé qu'Hincmar de Reims (v. 806-882) raconte dans sa vie de saint Remi - le circuit que Remi parcourt pendant la sieste de Clovis -, et qui semble inspirer aussi l'hagiographe d'un disciple supposé du

\footnotetext{
${ }^{17}$ fol. 71 (voir Die Urkunden der Merowinger, op. cit., p. 9, 1. 9-10) : primo nostre suscepte christianitatis atque subiugationis Gallorum anno nostre celsitudini tradidit et commendavit. Pour les passages dans les vies carolingiennes, voir supra n. 13.

${ }^{18}$ fol. 71-72 (voir Die Urkunden der Merowinger, op. cit., p. 9, 1. 12 et 20-21, p. 10, 1. 3, 4 et 7): peculiarem patronum nostrum; litteras manu nostra firmatas ipsi nostro patrono domno Iohanni dedimus ; peculiarem nostro patrono domno Iohanni ; pro ipso patrono nostro ac pro ipso loco sancto ; ipsa congregatio per tempora tanti patroni eiusque successorum. La recherche des différentes formes présentes de patronus dans la base de données des chartes bourguignonnes CBMA (Chartae Burgundiae Medii Aevi: http://www.artehis-cbma.eu/), montre qu'il est peu usité, avec seulement 54 occurrences dans 10278 chartes datant d'entre 500 et 1300 [patroni (11 fois), patronibus (4), patronique (1), patronis (4), patrono (7), patronorum (1), patronos (6), patronum (7), patronus (8)]. À part une occurrence dans un testament $\mathrm{du} \mathrm{VI}^{\mathrm{e}}$ siècle, le mot est employé seulement à partir du $\mathrm{X}^{\mathrm{e}}$ siècle (4 occurrences), puis aux XI (13 occurrences), pour désigner le saint patron d'un monastère.

${ }^{19}$ fol. 28r et 29r (nostrique singularis et summi patroni Iohannis iocundamur insigniis... Vnde nunciam ad summum nobis patronum Iohannem - homélie); fol. 45r (Tantum qui illi meruisti esse pedisecus, nobis digneris esse patronus - oraison); fol. 45v (R. O sacer Christi Iohannes tuorum patrone gloriosissime signorum in uirtuti dare audi uoces... - répons); fol. 46r (Plaudimus tanto quoties patrono debito certe rele uamur omnis quin et offensis ueniam meremur presule Christo... - hymnum ad nocturnos); fol. 57r (prout sufficit tenuitas ingenii nostri proprii patroni disserere laudem - BHL 4426, livre 1, chapitre 6).

${ }^{20}$ fol. 71v (voir Die Urkunden der Merowinger, op. cit., p. 9, 1. 8-9) : ...quia domnus Iohannes, clarus virtutibus, locellum suum in pago Tornotrinse sub regula Macharii ad habitationem monachorum constructum, quod Riomaus vocatur...

${ }^{21}$ fol. $5 \mathrm{v}$ et $58 \mathrm{r}$ : sub regulari tenore quam beatus Macharius aegyptiorum indidit monachis ministrare.

22 fol. 71v (voir Die Urkunden der Merowinger, op. cit., p. 9, 1. 13-14) : ut quantumcumque suo asino sedens una die circa locum suum nobis traditum et comendatum de nostris fiscis circuisset.
} 
saint évêque, saint Léonard de Noblat (dans le Limousin), qui comme dans le diplôme de Clovis pour Réôme, se fait confirmer par le roi l'aire qu'il sillonne sur le dos de son petit âne ${ }^{23}$. Le diplôme de Clovis pour Réôme, il faut le noter, est supposé avoir été passé à Reims (Actum Remis civitate). La figure du saint monté sur un âne pour délimiter le territoire de son monastère sera aussi associée à un autre saint abbé bourguignon, saint Seine, disciple de Jean de Réôme, fondateur de l'abbaye de SaintSeine en Côte d'Or, qu'on sculpte ainsi à la fin du XIII ${ }^{\mathrm{e}}$ siècle sur les bornes séparant les terres de son abbaye de celles de l'abbaye de Fontenay ${ }^{24}$.

Le diplôme de Clotaire est composé au regard de celui de Clovis : en souvenir et suivant l'exemple de son père (sicut dive memorie genitor noster Chlodoueus) ${ }^{25}$, Clotaire prend le monastère sous sa protection, lui accorde la libre élection de l'abbé ainsi que l'immunité ; il confirme la part du fisc que Clovis avait autrefois concédé en vue de l'entretien des moines. Comme dans le diplôme de Clovis, Jean est désigné dans son rôle de saint, patronus : il s'agit du monastère de saint Jean et du culte qu'on lui rend. Mais il s'adresse au disciple et successeur de Jean, l'abbé Silvestre ${ }^{26}$. En insistant sur le terme de successor, utilisé à cinq reprises, pour indiquer aussi bien la succession des rois que la succession des abbés, le diplôme dresse une généalogie double et croisée, royale et abbatiale (Clovis/Jean, puis Clotaire/Silvestre), de père en fils (Clovis/Clotaire), de maître à disciple (Jean/Silvestre). Il précise ainsi la geste des origines en posant, et en choisissant délibérément, des jalons affirmés. Ce parti pris marque un changement important par rapport aux récits hagiographiques, y compris la

\footnotetext{
${ }^{23}$ Pour la bibliographie et une analyse fine du rapport entre ces deux vies, voir M.-C. IsAÏA, Remi de Reims. Mémoire d'un saint, histoire d'une Eglise, Paris, 2010, p. 704-712. Hincmar de Reims, Vita Remigii episcopi Remensis, éd. B. KRUSCH, MGH Scriptores rerum merovingicarum, III, p. 306-307, ch. 17 : Unde, suadente religiosissima regina et petentibus locorum incolis, qui incolis, qui multiplicibus exeniis erant gravati, ut quod regi debebant aecclesiae Remensi persolverent, rex sancto Remigio concessit, ut, quantum circuiret, dum ipse meridie quiesceret, totum illi donaret. Sanctus autem Remigius per fines, quae manifestissime parent, pergens, signa sui itineris misit... Suirgente interea rege a somno meridiano, reversus est ad eum sanctus Remigius, et omnia que ambitus circuitionis illius continuit ei precepto suae auctoritatis rex donavit [BHL 7152-64 - avant 882]. Vita Leonardi Confessoris Nobiliacensis, éd. B. KRUSCH, MGH Scriptores rerum merovingicarum, III, p. 397-398, ch. 5 : Ad haec denique beati viri verba catholicus rex respondit: 'Sacratissimae voluntati tuae, homo Dei et amice religionis, habundanter satisfacere possum, quia sub cartulari praecepto tradam tibi istud nemus totum'. Cui beatus Leonardus: 'Non totum', inquid, 'serenissime rex, accipiam, sed partem, postquam exinde tuam sentio largiendi voluntatem'; et adiecit: 'Quantumcumque igitur ex hac silva cum meo asello peregrare potero, tantum et nom amplius a tua celsitudine mihi concedi exopto'. Quod rex sub regali decreto adimplevit, et quicquid ipse circuitione sui aselli comprehendisset, sicut idem vir sanctus expetierat, lapideis terminis concludi praecepit. Non expedit igitur litteris conscribere, quantum telluris ipse una nocte equitando valuit concludere; satis quippe habetur adhuc in recordatione, quidquid comprehensum sit ex beati viri circuitione [BHL 4862 - premier tiers du $\mathrm{XI}^{\mathrm{e}}$ siècle].

${ }^{24}$ Saint Seine est cité dans le manuscrit 1, aux feuillets 11v, 12r, 68r (BHL 4426). Sur les «grandes bornes » du bornage de 1288, dont six de celles connues aujourd'hui portent la gravure de saint Seine sur un âne d'un côté et celle de saint Pierre tenant une clé de l'autre ; voir A. CoutiER, M. MANGIN, «Sur les pas de Saint-Seine, les bornages médiévaux autour des Sources de la Seine », Mémoires de la Commission des Antiquités de la Côte d'Or, 40 (2002-2004), p.97-132, en particulier p. 109-114 et figures 28 à 37. Voir aussi Sculpture médiévale en Bourgogne: collection lapidaire du Musée archéologique de Dijon, M. JAnNET-VAllat, F. Joubert, Dijon, EUD, 2000, p. 78-79 (n 12 «Borne délimitant les territoires des anciennes abbayes de Saint-Seine-1'Abbaye et de Flavigny »); A. FETU, « Rapport sur les bornes délimitant le territoire de l'ancienne terre abbatiale de Saint-Seine », Mémoires de la Commission des Antiquités de la Côte d'Or, 13 (1895-1900), p. 5-7.

${ }^{25}$ fol. 73r (voir Die Urkunden der Merowinger, op. cit., p. 49, 1. 6).

${ }^{26}$ fol. 73r (voir Die Urkunden der Merowinger, op. cit., p. 49, 1. 6, 8-9, ) : monasterium domni patronis nostri Iohannis; ita et nos venerabilem Siluestrum, abbatem ipsius et domni Iohannis, nostri generis peculiaris patroni et oratoris, discipulum ac successorem, sub nostra nostrarumque regum successorum emunitate et defensione recepimus et revocamus.
} 
$B H L$ 4426, qui laissent dans le vague la relation de Jean, puis de Silvestre, avec les rois des Francs.

\section{Renovatio monastique et chantiers scripturaires}

Parmi les nombreuses hypothèses avancées sur l'époque et le contexte de la confection de ces actes, Theo Kölzer a sans doute raison de les placer au moment de la réforme monastique des alentours de l'an mil, sous l'abbatiat d'Heldric à Réôme (1003-1010). Disciple de Maïeul de Cluny, Heldric est un multi-abbé réformateur, ayant dirigé à la fois Flavigny, Saint-Germain d'Auxerre et Saint-Jean de Réôme. Dans un article de 1981, en développant une remarque de Robert-Henri Bautier sur le caractère suspect de deux diplômes de Charles le Chauve, Yves Sassier a montré l'entreprise de création de diplômes passant pour émaner des rois carolingiens à Saint-Germain et à Flavigny sous la direction d'Heldric ${ }^{27}$. Le même type d'entreprise à Réôme semble une hypothèse vraisemblable. On peut probablement placer dans ce contexte de renovatio monastique d'autres réalisations scripturaires des alentours de l'an mil et de la première moitié du $\mathrm{XI}^{\mathrm{e}}$ siècle, comme le manuscrit 1 de Semur-en-Auxois ou encore le livret hagiographique, inséré dans le manuscrit Reginensis latinus 493 de la Bibliothèque Vaticane (fol. 80r-135v) provenant de Saint-Germain d'Auxerre ${ }^{28}$, contenant la vie de Maïeul de Cluny et le dossier hagio-liturgique de Jean de Réôme inspiré du manuscrit de Semur ${ }^{29}$.

Le mouvement qui revisite les diplômes royaux pour les créer ou les recréer est plus large que les entreprises liées au multi-abbatiat d'Heldric. Plusieurs études ont pu déceler la fabrication de diplômes 'carolingiens' dans les églises et communautés d'Autun entre le $\mathrm{X}^{\mathrm{e}}$ et le $\mathrm{XI}^{\mathrm{e}}$ siècle. Selon l'hypothèse d'Olivier Bruand, le « minicartulaire » réunit vers la fin $\mathrm{du} \mathrm{X}^{\mathrm{e}}$ siècle pour l'abbaye féminine de Saint-Andoche, conservé aux Archives départementales de la Saône-et-Loire (H 675), où a été inséré un

\footnotetext{
27 Y. SASSIER, «Quelques remarques sur les diplômes d'immunité octroyés par les Carolingiens à l'abbaye Saint-Germain d'Auxerre », Bibliothèque de l'Ecole des Chartes, 139 (1981), p. 37-54 (repris et remanié dans ID., «Exemption monastique et remaniements textuels : les diplômes d'immunité octroyés par les carolingiens à l'abbaye Saint-Germain d'Auxerre », Structures du pouvoir, royauté et res publica (France IX $X^{e}-X I I^{e}$ siècle), Rouen, 2004, p. 93-109); R.-H. BAUTIER, Recueil des actes d'Eudes, roi de France (888-898), Paris, 1967 (Chartes et diplômes), n 11, p. 53 et n. 1. Constance Bouchard, éditrice des plus anciens actes de Flavigny, réfute Yves Sassier en considérant le diplôme de Charles le Chauve pour Flavigny de 849 comme authentique, mais cette réfutation ne repose sur aucune discussion de sa démonstration ou de son hypothèse, et se base seulement sur un rapprochement qu'il aurait fait du diplôme avec l'acte de fondation de Cluny, chose très étonnante car il n'est jamais question de cet acte dans l'article. Peut-être confond-elle l'acte de fondation avec l'acte d'exemption de Cluny de 998-999 ? [C. BritTAin Bouchard, The Cartulary of Flavigny, 717-1113, Cambridge (Massachusetts) (The Medieval Academy of America, Medieval Academy Books, 99), 1991, n 19 (p. 63)]. Sur l'abbaye SaintGermain d'Auxerre et l'abbatiat d'Heldric, voir aussi N. Deflou-LeCA, Saint-Germain d'Auxerre et ses dépendances ( $V^{e}-X I I I^{e}$ siècle), Saint-Étienne, 2010, p. 218-227.

28 A. Wilmart, Codices reginenses latini, Bibliotheca vaticana, 1937-1945, 2 vol. (Bibliothecae apostolicae vaticanae codices manu scripti recensiti), t. 2, p. 692-702.

${ }^{29}$ Le lien réalisé alors entre Maïeul et Jean de Réôme, et le rôle d'Heldric dans cette construction, ont été mis en avant par D. IOGNA-PRAT, Agni immaculati. Recherches sur les sources hagiographiques relatives à Saint-Maïeul de Cluny (954-994), Paris, 1988, p. 133-141, 287-301 : Heldric serait l'instigateur probable de la rédaction de la Vita Maioli (BHL 5179) entre 999 et 1010 et du Sermo de beato Maiolo dont une grande partie (1. 64-94) est empruntée à l'Homelia in nativitate sancti Iohannis, présente dans le manuscrit 1 de Semur-en-Auxois aux fol. 13v-14v. Il faut noter, par ailleurs, que cette homélie est utilisée aussi pour saint Trophime d'Arles (BHL 8318d) (cf. E. MAGNANI, « Trophimus, Dionisus, Regulus, Felicissimus... Listes et vies des premiers évêques d'Arles (IX ${ }^{\mathrm{e}}$-XIIe siècle) ", à paraître dans Provence historique, Actes du $57^{e}$ Congrès de la fédération historique de Provence (Marseille, octobre 2013) : La Provence, terre de cathédrales, dir. Y. CODOU et T. PECOUT).
} 
diplôme suspect de Charles le Simple ${ }^{30}$, ainsi que les pseudo-diplômes de Charles le Chauve pour la communauté canoniale de Saint-Symphorien réalisé peut-être autour de $993^{31}$, et de Charles le Gros pour le monastère de Saint-Martin à la fin du $\mathrm{X}^{\mathrm{e}}$ siècle $^{32}$, auraient été confectionnés sous l'épiscopat de l'évêque d'Autun, Gautier (976ca.1018) ${ }^{33}$. Ce prélat proche des milieux réformateurs clunisiens a, entre autres, confié la réforme de l'abbaye de Flavigny à l'abbé Heldric en 987/934.

Il faut aussi rappeler les rapports que l'abbaye de Réôme a entretenus avec l'abbaye de Saint-Bénigne de Dijon, dont le réformateur, Guillaume de Volpiano, abbé entre 990 et 1031, a aussi dirigé Moutiers-Saint-Jean entre 992 et $1002^{35}$. La Chronique de SaintBénigne, qui selon les dernières hypothèses de Karl Heidecker aurait été composée en 1053 , après la réalisation du cartulaire entre 1032 et $1053^{36}$, prend dans la vie de Jean de Réôme les personnages sur lesquels elle établit le récit de l'invention du corps du martyr Bénigne et de la fondation du monastère dijonnais au $\mathrm{VI}^{\mathrm{e}}$ siècle. C'est ainsi qu'au temps et qu'à l'initiative de l'évêque de Langres, Grégoire (506-539), une communauté aurait été installée auprès de la sépulture de Bénigne. Dans la vie de Jean de Réome, Grégoire est l'évêque qui oblige le saint à retourner à son monastère alors qu'il l'avait quitté pour séjourner incognito à Lérins ${ }^{37}$. Selon la chronique, le premier abbé de SaintBénigne, Eustade, était, comme saint Seine, un disciple de Jean de Réôme, et il était enseveli auprès de Bénigne tout comme les parents de Jean, Hilaire et Quiete ${ }^{38}$. L'un

\footnotetext{
${ }^{30}$ O. BRUAND, Les origines de la société féodale. L'exemple de l'Autunois (France, Bourgogne), Dijon, 2009, p. 18-19 ; I. FEES, « Drei Urkunden des Bischofs Jonas von Autun und die (angebliche) Synode von Saint-Geosmes », Deutsches Archiv für Erforschung des Mittelalters, 51/2 (1995), p. 376-403 ; C. BRÜHL, « Die Urkunde Karls des Einfältigen und Rudolf von Westfranken für das Nonnenkloster Saint-Andoche zu Autun », dans ID., Aus Mittelalter und Diplomatik, Gesammelte Aufsätze 2, Hildesheim, Munich, Zurich, 1989, p. 838-850 (repris de «Eine Fälschung auf den Namen Karls des Einfältigen für Nonnenkloster Saint-Andoche zu Autun », Historisches Jahrbuch, 91 (1971), p. 384-393. L'expression «mini-cartulaire» est de Nathalie Verpeaux, que je remercie pour les informations transmises sur ce dossier (Saint-Andoche et Saint-Jean-le-Grand : des religieuses à Autun au Moyen Âge, Thèse de doctorat de l'université Paris 1 Panthéon Sorbonne, 2009).

31 I. FEES, "Die Urkunde Karls des Kahlen für das Kloster Saint-Symphorien zu Autun: eine Fälschung ", Francia, 24/1 (1997), p. 65-82 ; O. BRUAND, Les origines de la société féodale, op. cit., p. 14 ; ID., "Autour des actes 2 et 4 du cartulaire de Saint-Symphorien d'Autun ou du bon usage de la falsification pour défendre un temporel monastique », André Déléage (1903-1944). Actes du colloque de Cluny, 3-5 septembre 2003, éd. A. Guerreau, D. Mehu, et coll. I. Vernus, Annales de Bourgogne, 831/3 (2011), p. 197-210 [je remercie l'auteur de m'avoir communiqué son article avant sa parution].

${ }^{32}$ O. BRUAND, Les origines de la société féodale, op. cit., p. 15-18.

${ }^{33}$ O. BRUAND, «L'évêque Gautier d'Autun. Un prélat faussaire ? », dans La foi dans le siècle. Mélanges offerts à Brigitte Waché, éds. H. GuILlemain, S. TisOn, N. VIVIER, Rennes, 2009, p. 121-132.

${ }^{34}$ N. Bulst, Untersuchungen zu den Klosterreformen Wilhelms von Dijon (962-1031), Bonn, 1973, (Pariser Historische Studien 11), p. 61 ss. Voir C. BRITTAIN BOUCHARD, The Cartulary of Flavigny, op. cit., n 28, p. 82-85 (mars 992).

${ }^{35}$ N. BuLST, Untersuchungen zu den Klosterreformen Wilhelms von Dijon, op. cit., p. 27.

${ }^{36}$ K. HEIDECKER, « Le cartulaire et la chronique de l'abbaye Saint-Bénigne de Dijon : le manuscrit 591 de la Bibliothèque municipale de Dijon » (à paraître dans Productions, remplois, mises en registre : la pratique sociale de l'écrit à travers la documentation médiévale bourguignonne, dir. E. MAGNANI, M.-J. GASSE-GRANDJEAN). Les actes de Saint-Bénigne de Dijon ont été édités dans Chartes et documents de Saint-Bénigne de Dijon, prieurés et dépendances, des origines à 1300, tome $1: \mathrm{VI}^{\mathrm{e}}-\mathrm{X}^{\mathrm{e}}$ siècles, R. FoLZ, J. MARILIER (éds.), Dijon, 1986 (Analecta Burgundica); Chartes et documents de Saint-Bénigne de Dijon, prieurés et dépendances, des origines à 1300, G. CHEVRIER, M. CHAuME (éds.), tome 2 : 9901124, Dijon, 1943 (Analecta Burgundica).

${ }^{37}$ Semur-en-Auxois, Bibliothèque municipale, ms. 1, fol. 5r, 56r.

${ }^{38}$ Chronique de l'abbaye de Saint-Bénigne de Dijon, F. Bougaud, J. GARniER (éds.), Paris, 1875 (Analecta divionensia 9), p. 10-15. Ces emprunts factuels à la vie de Jean de Réôme n'ont pas été signalés par C. Dahlmann, "Untersuchungen zur Chronik von St. Bénigne in Dijon», Neues Archiv der Gesellschaft für ältere deutsche Geschichtskunde, 49 (1932), p. 281-331.
} 
des cinq autels de la crypte de Saint-Bénigne était dédié à saint Jean, à saint Seine et à saint Eustade ; l'abbaye conservait aussi la moitié du corps de saint Grégoire, l'évêque de Langres ${ }^{39}$.

Les consonances entre les deux abbayes ne se limitent pas aux emprunts textuels et peuvent avoir joué aussi dans leur façon de concevoir la production de diplômes. En fait, à l'instar de Réôme, à Saint-Bénigne on a aussi fabriqué un pseudo-diplôme mérovingien, peu après 1031 selon Theo Kölzer ${ }^{40}$. Il est connu par sa transcription dans le cartulaire de Saint-Bénigne (entre 1032 et 1053) et mentionné dans la chronique ${ }^{41}$. Il s'agit d'un plaid où le roi Clotaire (III) règle en faveur de l'abbé de Saint-Bénigne un différend au sujet de la villa Larrey-sur-Ouche (664/65, 24 octobre [à Mâlay-le-Petit]). En fait, tout en mentionnant un (ou deux ?) diplôme antérieur (perdu) du roi Gontran $(561-592)^{42}$, et, chose inhabituelle, sans nommer l'adversaire de l'abbaye, ce document est en grande partie conçu sous la forme d'une notice relatant un règlement de conflit caractéristique du $\mathrm{XI}^{\mathrm{e}}$ siècle. En acceptant la reconstitution du cartulaire proposée par Karl Heidecker, on observe qu'un rôle important est attribué à cette notice, car elle ouvre le cartulaire, placée en tête d'un quaternion réunissant neuf actes royaux en faveur de l'abbaye (aujourd'hui le cahier 11 du manuscrit 591 de la Bibliothèque municipale de Dijon) ${ }^{43}$. À l'inverse des copies figurées des diplômes carolingiens qui distinguent ce cartulaire, dans la transcription des actes mérovingiens ce sont seulement quelques « reminiscences ${ }^{44}$, d'ordre graphique et performatif ${ }^{45}$, qui sont employées et émaillent ainsi le rendu visuel des copies. Dans l'acte de Clotaire III pour Saint-Bénigne, le chrismon, les litterae elongatae de la première ligne, les majuscules dans l'eschatocole où entre la date et le signe de validation l'espace laissé en blanc fait allusion à la place réservée pour le sceau, sont des procédés graphiques qui rappellent ceux utilisés dans les diplômes de Clovis et de Clotaire I ${ }^{\mathrm{er}}$ pour Saint-Jean de Réôme dans le manuscrit 1 de Semur-en-Auxois. Ici, à part l'invocation en lettres allongées au début du diplôme de Clovis, l'accent est mis sur les protocoles finaux, parés de monogrammes royaux ainsi que de signes de recognition, des ruches inventées pour l'occasion (fig. 5 et 6).

\footnotetext{
${ }^{39}$ Chronique de l'abbaye de Saint-Bénigne, op. cit., p. 142-143. On retrouvera la bibliographie relative à l'abbaye Saint-Bénigne de Dijon dans C. MARIno Malone, Saint-Bénigne et sa rotonde. Archéologie d'une église bourguignonne de l'an Mil, Dijon, 2008. Sur les abbés voir H. Hellerström, 'Historia huius loci'. Studien zum Bild von Abt und Mönch in der Chronik von Saint-Bénigne, München, 1977.

${ }^{40}$ Die Urkunden der Merowinger, op. cit., t. 1, n 103, p. 264-268 (daté de 664/65).

${ }^{41}$ Dijon, Bibliothèque municipale, ms. 591, fol. 69v-70 ; Chronique de l'abbaye de Saint-Bénigne, op. cit., p. 61-62.

${ }^{42}$ Die Urkunden der Merowinger, op. cit., t. 2, Dep. 67, p. 527-528.

${ }^{43}$ Dans la chronique de Saint Bénigne, la mention de cet acte précède la transcription d'une bulle du pape Sergius I ${ }^{\text {er }}$ (687-701) garantissant le droit de sépulture au monastère ; cette bulle est aussi une création de la première moitié $\mathrm{du} \mathrm{XI}^{\mathrm{e}}$ siècle rédigée sur l'envers du papyrus d'une lettre pontificale de la fin du $\mathrm{X}^{\mathrm{e}}$ siècle (Chronique de l'abbaye de Saint-Bénigne de Dijon, op. cit., p. 61-62 (voir aussi p. 15). Sur ce papyrus voir, P. GRAS, "Une bulle de plomb du pape Jean XV (995) », Bibliothèque de l'Ecole des Chartes, 122 (1964), p. 252-256) et H. APPELT, « Die falschen Papsturkunden des Kloster St. Benigne de Dijon », Mitteilungen des Instituts für österreische Geschichtsforschung, 51 (1937), p. 249-312.

${ }^{44}$ L'expression est de Theo Kölzer, Die Urkunden der Merowinger, op. cit., t. 1, n 103, p. 265-66. Voir aussi K. HEIDECKER (art. cit.).

${ }^{45}$ Sur le caractère performatif des cartulaires enluminés, doir R. A. MAXWELL, « Sealing Signs and the Art of Transcribing in the Vierzon Cartulary », The Art Bulletin, 81/4 (1999), p. 576-597.
} 


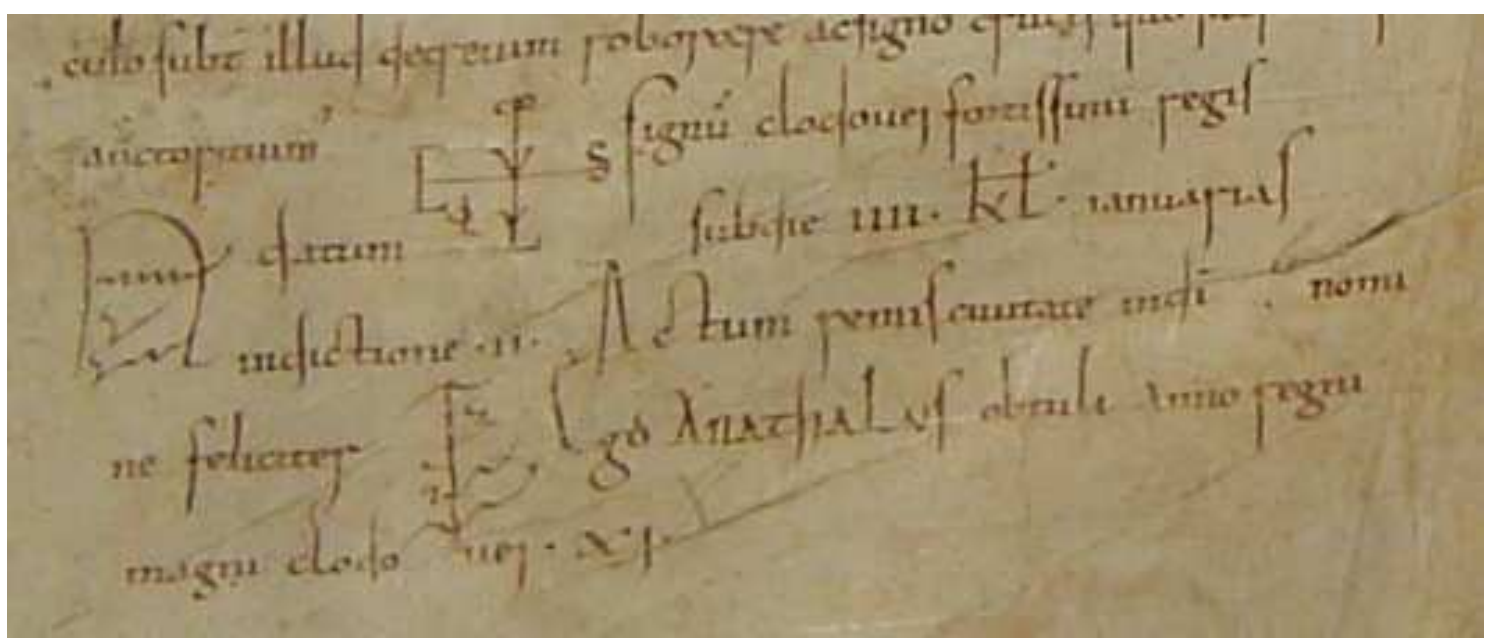

Fig. 5 : Semur-en-Auxois, Bibliothèque municipale, ms. 1, fol. 72v (protocole final du pseudo-diplôme de Clovis I ${ }^{\text {er }}$ pour Saint-Jean de Réôme)

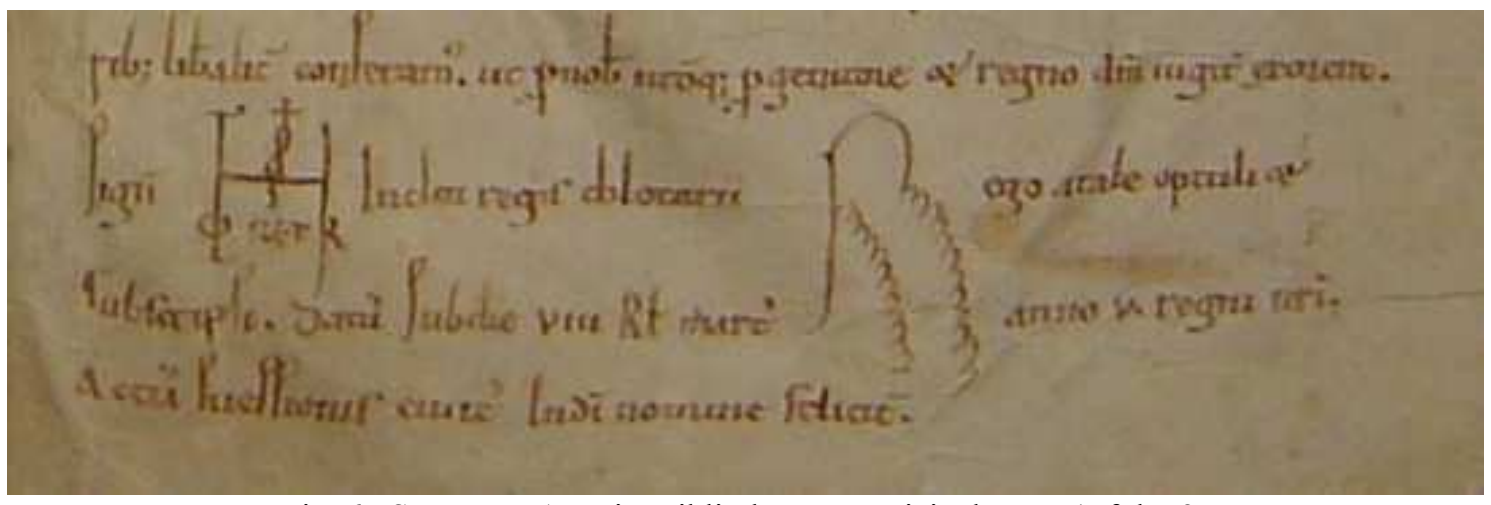

Fig. 6 : Semur-en-Auxois, Bibliothèque municipale, ms. 1, fol. $73 \mathrm{r}$ (protocole final du pseudo-diplôme de Clotaire $\mathrm{I}^{\mathrm{er}}$ pour Saint-Jean de Réôme)

Entre le $\mathrm{X}^{\mathrm{e}}$ et le $\mathrm{XI}^{\mathrm{e}}$ siècle, la réforme des anciens monastères bourguignons est un moment de renouveau profond, qui n'affecte pas seulement la vie consacrée, liturgique, menée à l'intérieur des communautés, mais engage également une redéfinition de la place et de l'action des monastères et des moines dans la société seigneuriale. Les chantiers scripturaires de cette période, comme celui qu'on peut essayer de restituer autour du manuscrit 1 de Semur-en-Auxois, rendent compte, à leur manière, de ce double moteur. La création et l'invention passent à la fois par l'appréhension active de ce qui est connu et existe déjà, et par sa transformation à la suite d'un jeu subtil de déplacements et de renvois. Ces émaillages de textes, de signes, d'images, lancent une dynamique qui se prolonge et se prête, au besoin, à des nouvelles actualisations, en se servant (et en étant servis par) d'un support versatile, le codex, qui les rend possibles tout en les déterminant à son tour. Les concepteurs, réviseurs, utilisateurs du livret hagio-liturgique de saint Jean de Réôme et des diplômes de Clovis et de Clotaire dialoguent, échangent, composent, avec des mots et des regards, comme une manière de peser sur l'insertion de leur communauté dans un monde en mouvement. 